\title{
Enlarged Ingroup Effect: How a Shared Culture Shapes In-Group Perception
}

\author{
Paola Rigo $^{1}$, Bindiya L. Ragunath ${ }^{2}$, Marc H. Bornstein ${ }^{3,4}$, Gianluca Esposito ${ }^{2,5,6^{*}}$
}

1 Department of Developmental 78 Psychology and Socialisation, University of Padua, Padua, Italy

2 Psychology Program, School of Social Sciences, Nanyang Technological University, Singapore, Singapore

3 Institute for Fiscal Studies, London, United Kingdom;

4 Eunice Kennedy Shriver National Institute of Child Health and Human Development, Bethesda, USA

5 Lee Kong Chian School of Medicine, Nanyang Technological University, Singapore, Singapore

6 Department of Psychology and Cognitive Science, University of Trento, Rovereto, Italy

Correspondence to gianluca.esposito@ntu.edu.sg

ABSTRACT: Increasing levels of migration and constant redefinition of a 'sense of belonging' characterize modern societies. Thus, social perception of people from different ethnicities as in-group or out-group members is influenced by a shared culture that might go beyond ethnicity. Using functional magnetic resonance imaging, we aim to study how sharing a common culture changes the social perceptions of in-groups and out-groups. We presented same- and different-race faces to young adults living in an integrated multicultural society. Same- and different-race faces were primed by images of environmental context that promotes identification with the participants' ethnicity or a common shared culture.

We found that same and different-race faces recruit similar brain networks only when associated with an environmental context, which promoted identification with a common shared culture. These results support a possible emergent phenomenon in multicultural societies that we call enlarged multi-ethnic in-group effect, which may form the basis of a potential new way to categorize oneself and others in terms of membership.

KEYWORD: same race faces, different race faces, cultural and ethnic context, enlarged in-group effect, face categorization

\section{Introduction}

Due to rapid urbanization and globalization resulting in increasing levels of migration (Kærgård, 2010), many countries today have residents of various ethnic backgrounds (Dijkstra, Geuijen, \& De Ruijter, 2001; Holtug, 2016; Mahmud \& Jahan, 2013; Keong, 2013). A challenge for such multicultural societies is to understand complex social interactions to ensure social cohesion and harmony (Dijkstra et al., 2001; Rosenthal \& Levy, 2010). People make sense of the complex social world through social categorization (Derks, Stedehouder, \& Ito, 2014; Kaul et al., 2012; Van Bavel \& Cunningham, 2010). People perceive themselves and other people they encounter as members of social categories based on salient social categories, such as, ethnicity, gender, age, and nationality, (Derks et al., 2014; Van Bavel \& Cunningham, 2010; Kurzban, Tooby, \& Cosmides, 2001), some of which are automatically encoded (Kaul et al., 2012), and which shape the early processing of visual features of others (Derks et al., 2014).

In multicultural societies, the negative aspects of ethnic diversity are addressed at communal and political levels (Gijsberts, Van Der Meer, \& Dagevos, 2011; Keong, 2013). Rosenthal and Levy (2010) summarized a multicultural approach that proposes social categorization, such as race and ethnicity, should be given attention and understood in order to develop knowledge and respect for other groups. According to these authors, multiculturalism is characterized by three central aspects: acknowledgment of differences between ethnic groups; appreciation of each group's contributions to society; and emphasis on groups maintaining their own culture and traditions (Rosenthal \& Levy, 2010). Enhanced social interaction and integration can lead to the development of a common shared culture across multi-ethnic groups that all members can identify with, in addition to their ethnic identity.

On one hand, there is a tendency to divide the social world into 'us' and 'them' (Shkurko, 2012; Van Bavel \& Cunningham, 2010), and on the other, ingroup and out-group categorization can be fluid, context-dependent, and mediated by its relevance for self-definition in terms of personal or social identity (Turnet et al., 1994). In fact, people can be members of various groups simultaneously or categorize others as in-group and out-group members depending on the more relevant categorization in the ongoing social situation. For example, some categorization criteria include the individual's goals, expectations, desires, and beliefs important for self-identification (Hehman 
et al, 2011; Katsumi \& Dolcos, 2018; Wheeler and Fiske, 2005; Turner et al., 1994, Cheon \& Esposito, 2020). Social perceptions of people as in-group or out-group members are influenced by ethnicity, formal and informal social roles that drive relations between minority and majority ethnic groups in daily life, as well as many other psychological factors (i.e., facial features, emotional reactions, and cognitive aspects) (Elfenbein \& Ambady, 2002; Soto \& Levenson, 2009).

Faces can communicate information quickly and efficiently (Hugenberg \& Wilson, 2013; Caria et al., 2012; Esposito et al., 2014; Esposito et al. 2015; Venturoso et al. 2019). Research suggests that neural responses to faces present an internal reflection of in-group and out-group perceptions. Much research on face perception and recognition were aimed at understanding in-group and out-group perceptions (e.g., Bavel, Parker, \& Cunningham, 2008), mainly because faces provide important social cues critical to social categorization (e.g., sex, race, age), emotional evaluation, and intentions. Investigating neural responses to in-group and outgroup social perceptions has gained ample attention (for review see Adolphs, 2001; Kubota, Banaji, \& Phelps, 2012; Shkurko, 2012). Neural substrates involved in out-group social cognition using faces include visual attention networks such as the fusiform gyrus (FG); amygdala in self-regulatory processes; and social areas such as anterior cingulate cortex (ACC), temporoparietal junction (TPJ), and the superior temporal sulcus (STS).

Research suggests intergroup conflict and bias can be reduced by shifting categorizations such that former out-group members are included in the ingroup (Gaertner \& Dovidio, 2000). Members who belong to double in-groups (e.g., same race/same university) are preferred over members of partial ingroups (e.g., same race/different university), which in turn are preferred over members of double outgroups (e.g., different race/different university) (Crisp \& Hewstone, 1999; Hehman et al., 2011). Furthermore, EEG findings showed that in facerecognition of double in-group members, compared to partial in-group or double-outgroup, participants' brain responses were sensitive to contextual information beyond ethnicity (e.g., Hehman et al., 2011). Double in-group faces evoke a larger event-related potential (ERP) component, followed by faces of partial in- groups, indicating more significant neural processing in the attention networks (Hehman et al., 2011).

In this study, we propose that face processing of same-race and other-race ethnicities will differ based on the cultural context shared by people. Recruited participants were citizens living in Singapore, a multicultural social environment where citizens identify with their ethnic as well as their national identity, and whose multicultural stance accords with the three central aspects in multiculturalism postulated by Rosenthal \& Levy (2010) (Salleh, 2017; Straits Times, 2017).

In this study, we investigated the effect of ethnic and shared cultural contexts on same-race and otherrace face perception in young adults of Chinese ethnicity. Participants underwent fMRI scanning while viewing same-race and other-race faces preceded by an ethnic context (e.g., Chinese temple before a Chinese face or Taj Mahal before an Indian face), or a shared cultural context pictures (e.g., famous Singapore national monument/building).

Since our participants live with people of specific ethnicities with whom they share everyday life (typical ethnicities), we considered two types of out-groups in the present study: atypical and typical other-race faces. We postulated that neural responses to other- (out-group) and same-race faces (in-group) faces would differ in environmental contexts that promote identification with one's ethnicity consistent with literature. In contrast, identifying with a shared culture (i.e., cultural context) would result in similar neural responses to other- and same-race faces, potentially indicating an automatic and spontaneous sense of membership to a multiethnic group. When primed by cultural context, we argue that typical other-race faces, but not atypical other-race faces (shared culture or enlarged in-group effect), and same-ethnicity faces (same-race in-group effect) will recruit similar brain regions.

We first hypothesize that typical other-race faces (Indian), when preceded by ethnic context (race effect), increase the brain activation in regions typically associated with out-group face categorizations (fusiform gyrus and visual cortex), self-regulatory and approaching/avoidant behaviors (amygdala, IFG). As a control condition for out-group faces, we included non-typical ethnic faces (Arabic, Caucasian).

In contrast, we then postulated that both typical other-race faces and same-race faces, when 
preceded by shared cultural context as opposed to ethic context, would increase brain activations in regions associated with personal identity, self, empathic aspects, and pro-social behaviors. Specifically, the midline brain structures (PCCCuneus MPFC), superior-inferior parietal cortex, lingual gyrus, STG, and inferior frontal cortex (Xu et al., 2009; Molenberghs 2013; Scheepers et al., 2013; Katsumi \& Dolcos, 2017). Findings will be discussed with relevance to existing literature along with implications.

\section{Methods}

\section{Participants}

A total of 48 participants (50\% females) aged between 21 to 26 years $(M=22.81, S . D .=1.17)$ took part in this study. All participants were recruited from Nanyang Technological University (NTU), Singapore. All participants were Singaporean Chinese who had not travelled out of Singapore for more than 2 months over the past 6 months from the time of their experimental session. They were also screened to ensure right-handedness, normal or corrected-tonormal vision, and no history of psychological or neurological disorders. No female participants were pregnant. Participants had to refrain from alcohol, nicotine, and caffeine consumption 24 hours prior to the scan session. This study was approved by the NTU IRB (Protocol 2017-01-029). Written informed consent was obtained from all participants. All data are available at this URL: https://doi.org/10.21979/N9/IC672L

\section{Experimental Task}

To measure effects of context (common shared cultural context (CulCon) or ethnic context (EthCon), on brain responses to in-group faces (IF) and out-group faces $(O F)$ ), we asked participants to view images of faces primed with CulCon or EthCon during the fMRI scan. Since the participants were all Singaporean Chinese, ethnically, IF consisted of Chinese faces. OF consisted of a common typical ethnicity present in Singapore, Indian faces, and nontypical ethnicities of Singapore, Arab and Caucasian faces. Faces of four ethnicities were categorized into typical ethnicities (Chinese and Indian faces) of Singapore and non-typical ethnicities (Arabic and Caucasian faces). Typical ethnicities were further categorized into IF (Chinese faces) and OF (Indian faces). CulCon comprised famous buildings/monuments of Singapore (e.g., Merlion, Esplanade) and EthCon contained famous buildings relevant to each ethnicity (e.g., Taj Mahal for Indian ethnicity, Chinese temple for Chinese ethnicity, Eifel Tower for Caucasian ethnicity, and the Sheik Zayed Mosque for Arabic ethnicity). All images were presented in grayscale. All faces were female and were masked with a grey round window so that only facial features were visible (no hair or neck were visible). Each trial started with a fixation cross displayed between $7-10$ seconds, followed by a contextual picture (context condition) displayed for 2 seconds, then followed by an image of a face for 4 seconds (Figure 1). The task consisted of a total of 32 trials. The faces and their preceding contextual picture stimuli were randomized for each participant. The duration of the fixation cross was also randomized. To assess the effect of EthCon, only congruent EthCon to ethnic faces were considered (e.g., Indian faces only preceded by Taj Mahal, Caucasian faces only preceded by Eiffel Tower).
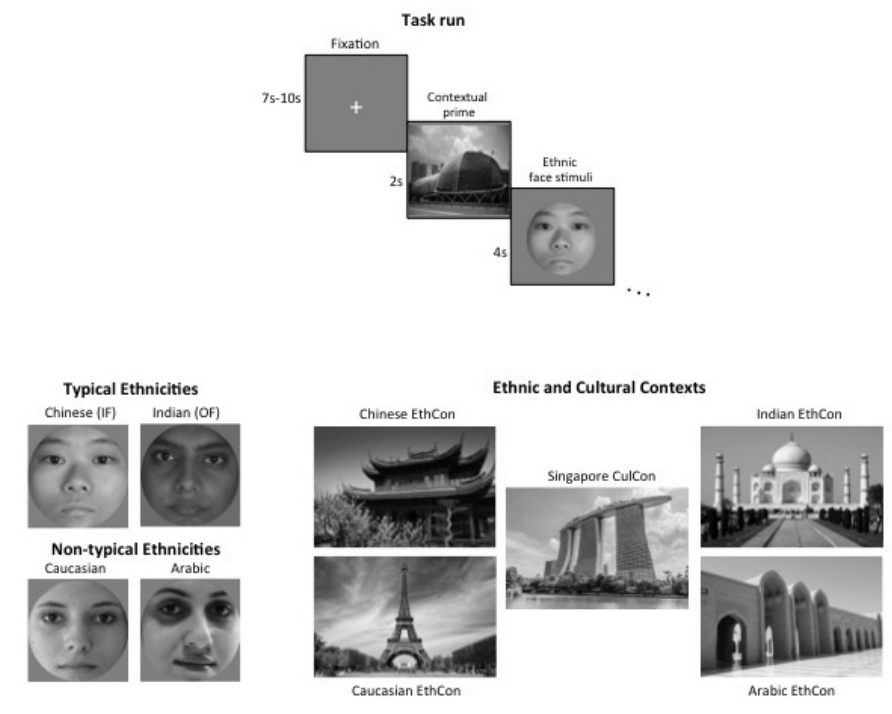

Figure 1. Task paradigm.

\section{Procedure}

Prior to their testing session, eligible participants were briefed about the study and underwent a MRI safety briefing before they were scanned. Participants were also reminded of the voluntary nature of the study. The experimental task took place during the fMRI scan, where the images were displayed on a screen, and participants viewed them through a mirror positioned at their eye level on the head coil. Participants were instructed to simply 
view the images displayed to them during the scan. Anatomical scans were also obtained. After the scan session, participants were debriefed about the specific aims of the study and were provided monetary reimbursement of $\mathbf{S} \$ 50$.

\section{MRI and fMRI Data acquisition}

Whole brain neuroimages were collected using a Siemens Magnetom Prisma 3-Tesla MRI Scanner with 64-channel head coil. A high-resolution T1-weighted MPRAGE sequence (192 slices; TR $2300 \mathrm{~ms}$; TI 900ms; flip angle 8 degrees; voxel size $1 \mathrm{~mm}$ ) was obtained to serve as an anatomical reference. Subsequently, functional images were obtained using a gradient Echo Planar Imaging (EPI) sequence with 36 axial slices (271 slices; slick thickness $3 \mathrm{~mm}$ with no inter-slice gap), and the following parameters: TR 2000ms; TE 30ms; flip angle 90 degrees; FOV 192 x 192 mm; voxel size $3 \mathrm{~mm}$; interleaved. External head restraint (i.e. neck padding) was used to minimize head movement during the scan.

\section{fMRI Data analysis}

fMRI analysis was conducted using Statistical parametric mapping

(SPM12; (http://www.fil.ion.ucl.ac.uk/spm/software/spm12) on the Matlab 2017b platform. Functional image preprocessing consisted of the following steps: Discarding the first two volumes of the functional time series, correcting for head movement, and then coregistering the $\mathrm{T} 1$ anatomical image to the mean of the realigned functional images carried out functional image preprocessing. Functional images were then normalized to the Montreal Neurological Institute (MNI) stereotaxic standard space and finally smoothed spatially (9-mm full-width half-maximum Gaussian kernel) and temporally (cut-off period 256 seconds). Analytic design matrices were constructed for each participant, which modeled the onsets and duration of the face image from each trial as epochs convolved with a hemodynamic response function.

General linear modeling (GLM) was performed to assess the effects of ethnic context (EthCon) and culture context (CulCon) amongst typical ethnicities in Singapore (Chinese and Indian faces) and non-typical ethnicities in Singapore (Arabic and Caucasian faces). A total of four conditions were modeled as separate regressors at individual level $\left(1^{\text {st }}\right.$ Level) analysis: typical ethnic faces in CulCon, typical ethnic faces in EthCon, non-typical ethnic faces in CulCon, and non-typical ethnic faces in EthCon. Specific weight vectors were denoted to derive contrast images for second-level (group) mixed effects analysis using a general linear model (GLM). The contrasts of interest were:

1.1 typical faces in CulCon vs. EthCon to investigate the effect of culture and ethnic contexts on typical ethnicities; and

1.2 non-typical faces in CulCon vs. EthCon to assess the effect of cultural and ethnic contexts on nontypical ethnic faces.

In the 2nd level random-effect analysis, one sample t-tests were conducted to assess group effects on the contrast images from the 1 st level. The t-tests indicated whether observed differences in the abovementioned contrasts (1.1 and 1.2) differed significantly from zero (Holmes and Friston, 1998) and allowed for inferences across participants.

A second GLM was performed to further assess the effect of cultural and ethnic context amongst IF and $O F$ of typical ethnicities. A total of four conditions were modeled as separate regressors; IF in EthCon, $O F$ in EthCon, IF in CulCon, and OF in CulCon. Specific weight vectors were denoted to derive contrast images for group level (2nd Level), mixedeffect analysis. The contrasts of interest were

2.1 IF in CulCon vs. EthCon to compare neural responses of in-group faces primed with cultural context to in-group faces primed with ethnic context;

2.2 OF in CulCon vs. EthCon to compare neural responses of out-group faces primed with cultural context to out-group faces primed with the ethic context;

2.3 OF vs. IF in CulCon to assess how neural responses to $O F$ and IF differed in the cultural context; and lastly,

2.4 OF vs. IF in EthCon to assess how neural responses to $O F$ and IF differed when primed with ethnic context.

Additionally, two other SPM(t) contrasts were created to assess how different the contrasts OF in CulCon vs. IF in CulCon (2.3) were from the contrast of OF in EthCon vs. IF in EthCon (2.4).

Finally, through the conjunction analysis, we assess the similarity between contrasts (2.3) and (2.4). 
For all analyses, a threshold significance of $\mathrm{p}<0.05$ was set for resulting SPM(t) with a cluster-wise $(\mathrm{k}=$ 10) family-wise error rate (FWE) correction for multiple comparisons (Logan \& Rowe, 2004). Whole brain analyses were performed.

\section{Results}

Neural Responses to Typical (1.1) and Nontypical (1.2) faces in Cultural compared to Ethnic Context

Comparing faces of typical ethnicities present in Singapore (Chinese and Indian) in the cultural (CulCon) versus the ethnic (EthCon) contexts, we found significant activated clusters as follows:

- Typical faces in CulCon > typical faces in EthCon: Higher activations in the right superior temporal sulcus (STS), left middle temporal gyrus (MTG), right supramarginal gyrus (SMG), right visual association area (VAA), left VAA extending to the lingual gyrus (LG), and the right primary sensory area (PSA) comprising of the postcentral gyrus (postCG).

- Typical faces in EthCon > typical faces in CulCon: Higher activation of the fusiform gyrus (FG) and middle occipital gyrus (MOG).

Comparing faces of non-typical ethnicities (Arab and Caucasian) in the cultural CulCon versus the EthCon contexts, we found significant activated clusters as follows:

- Non-typical faces in EthCon > non-typical faces in CulCon: the only activation seen was in the right fusiform gyrus (Table 1, Figure 2).

Peaks of all brain activations for these respective contrasts are summarized in Table 1. Refer to Figure 2 for the difference in activation patterns for faces of typical ethnicities and non-typical ethnicities in ethnic and cultural contexts.

\section{Neural Responses to In-group Faces in Cultural and Ethnic Contexts}

To investigate brain responses to in-group Chinese faces in cultural and ethnic contexts, we compared neural responses of in-group faces in the cultural context condition to in-group faces in the ethnic context condition; in-group Chinese faces (IF) in the cultural (CulCon) versus the ethnic (EthCon) context conditions (contrasts 2.1). We found significant activated clusters as follows:
- IF in CulCon > IF in EthCon: Significant activations of the right VAA comprising of the LG, right PSA comprising of the postCG, and rolandic operculum (RO), a part of the secondary somatosensory area was obtained.

- IF in EthCon > IF in CulCon: Higher activations in $F G$ and MOG. All activations were limited to the right hemisphere (Figure 3).

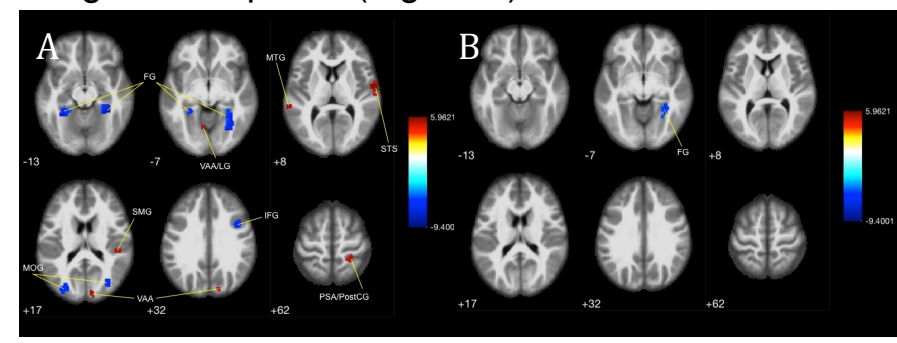

Figure 2. (A) Brain activations to typical ethnicities in Singapore in cultural versus ethic context (typical faces in CulCon > typical faces in EthCon), shows activation in multiple regions compared to (B) which shows activation only in the FG for non-typical ethnicities in cultural versus ethnic context (nontypical faces in CulCon > non-typical faces in EthCon).

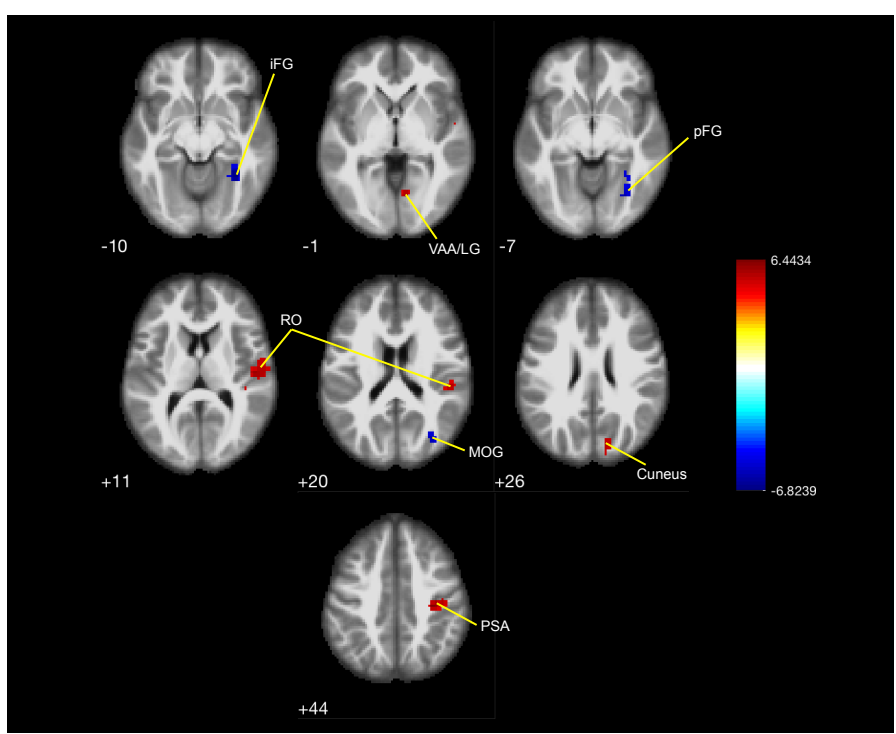

Figure 3. Brain activation of in-group Chinese faces in cultural context versus ethnic context (IF in CulCon $>$ IF in EthCon).

Neural Responses to Typical Out-group (typical other-race) Faces in Cultural and Ethnical Contexts

Neural responses of culturally primed out-group faces were compared to ethnically primed out-group faces; 2.2) OF in CulCon vs. EthCon, to understand the effect of context on out-group faces. Due to the lack of ample trials, as we have obtained this contrast from a larger task, and with a stringent FWE correction, no significant activation was found at 
$\mathrm{p}(\mathrm{FWE})<0.05$. However, with a less stringent uncorrected $p$-value 0.001 we found significant cerebral brain activation in (Table 3 ):

- OF in CulCon > OF in EthCon: Higher activation in the VAA, right FG, and the left postCG, a part of the somatosensory cortex.

\section{Preliminary contrasts for Difference and Similarities of Neural Responses to Out-group and In-group based on Context \\ Comparing Neural Responses of Out-group and In- group in Cultural Context}

If a common shared culture does drive an automatic and spontaneous sense of membership to a multiethnic group, then brain responses to out-group faces primed with culture, representative of an enlarged cultural in-group, should be similar to brain responses to in-group faces. Thus, any differences between outgroups and in-groups in the cultural context were assessed; 2.3) OF vs. IF in CulCon.

- OF in CulCon > IF in CulCon: We found no significant differences in brain responses to ingroup and out-group faces in the cultural context at $\mathrm{p}(\mathrm{FWE})<0.05$ (Figure 4A). Even at a less stringent uncorrected $p<0.001$, the only difference was a higher activation in the right $F G$ when participants viewed in-group compared to outgroup faces (see Table 4).

Comparing Neural Responses of Out-group and Ingroup in Ethnic Context

The contrast 2.4) OF vs. IF in EthCon was examined to compare how out-group and in-group faces were processed in the ethnic context.

- OF in EthCon > IF in EthCon: Higher activation of the right VAA extending to the LG, left LG, right sensory association area (SAA), right cuneus, RO a part of the secondary somatosensory area, left STG, and left SMG were found when processing out-group faces (see Table 5 and figure 4).

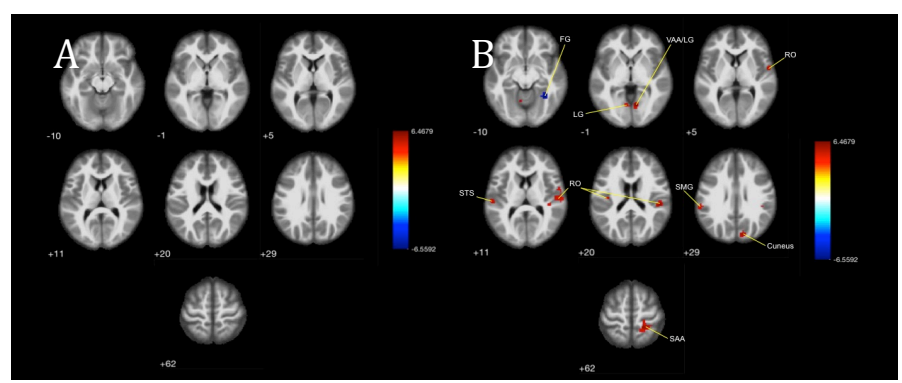

Figure 4. (A) No significant difference in brain activation when viewing out-group faces versus ingroup faces in the cultural context (OF in CulCon > IF in CulCon) suggesting an enlarged in-group based on cultural identity, which is not present in (B) when viewing out-group versus in-group faces in the ethnic context (OF in EthCon > IF in EthCon).

\section{The Difference and Similarities of Neural Responses to Out-group and In-group based on Context}

OF in CulCon vs. IF in CulCon differed from OF in EthCon vs. IF in EthCon. Similarities in neural responses of these contrasts were also assessed through conjunction analysis. Table 6 displays both similarities and differences in activations for the abovementioned contrasts. The cortical midline regions were significantly more active for the cultural context condition compared to the ethnic context condition. These regions were the left and right lingual gyrus, right precuneus and cuneus, and left precentral gyrus. On the contrary, the left and the right fusiform gyrus were significantly active for both cultural and ethnic context conditions.

\section{Discussion}

This study postulated that by identifying with a common shared culture among various racial ethnicities in multicultural societies, the neural responses to other- and same-race faces would be similar, indicating an automatic and spontaneous sense of membership to multi-ethnic groups (that we called enlarged cultural in-group effect). Specifically, using a modified priming paradigm, we investigated the neural response to out-group faces of typical ethnicities present in Singapore (Indian faces), nontypical ethnicities (Arab and Caucasian faces) and ingroup faces (Chinese) in the ethnic and in the (shared) cultural context conditions.

\section{More effort in processing out-group faces in ethnic context contrasted to cultural context}

When typical out-group faces (typical other-race face; Indian) were viewed in the ethnic as compared to the cultural context conditions, a high engagement of visual processing and social cognitive regions was found. Specifically, the ethnic context increased the cerebral activation in the VAA, implicated in enhanced visual processing (Rosen et al., 2018); in the lingual gyrus, associated with outward-directed social and contextual focus (Colton et al., 2013); and in the left postCG, part of the somatosensory cortex (Chan \& Baker, 2015; Green et al., 2018), involved in 
mentalizing networks, social emotions and social perception (Keysers et al., 2010; Sugranyes et al., 2011; Bernhardt \& Singer, 2012; Schaefer et al., 2013; Valchev et al., 2016). Activation in the FG was also present. The $F G$ is implicated in top-down and bottom-up perception of visual input. Bottom-up perception includes higher FG activation to racial ingroup faces than out-group faces (Guassi Moreira et al., 2016; Molenberghs, 2013; Shkurko, 2012; Van Bavel et al., 2008). The top-down modulation of $F G$ in social perception involves indexing socially significant perceptual input according to preexisting schemas of the social environment (Shkurko, 2012). Thus, FG is considered of the first few regions to respond to social faces and may represent the top-down process involved in explicit social categorization in response to the social context (Shkurko, 2010). Therefore, activations of higher visual processing and social cognitive regions for out-groups in the ethnic context may indicate more effort in processing the out-group faces in the ethnic context.

Brain regions characteristic of out-group activation such as the amygdala (Derntl et al., 2009; Freeman et al., 2010; Molenberghs, 2013; Shkurko, 2010; Van Bavel et al., 2008), ACC (Cunningham et al., 2004; Kaplan et al., 2007; Knutson et al., 2007), or insula (Falk et al., 2012; Kaplan et al., 2007; Phelps et al., 2000) were not found in the present study. Amygdala is implicated in social categorization and implicit racial bias (Cunningham et al., 2004; Shkurko, 2010). Cunningham et al. (2004) reported amygdala activation when faces were presented before conscious processing $(30 \mathrm{~ms})$ could occur. Such activation was absent when faces were presented for a longer duration $(525 \mathrm{~ms})$, allowing for conscious processing where implicit racial bias would be regulated. Therefore, a possible reason for the lack of amygdala activation could be because the face stimuli were presented for 4 seconds, allowing for any regulation of racial bias. If this stands true for the current study, then ACC activation should have been obtained, to consciously regulate out-group bias, similar to that of Cunningham et al.'s findings (2004). However, no activation in the ACC was found here. Zuo and Han (2013) reported that neural activity in the pain matrix, including the anterior cingulate cortex and anterior insula, did not differ for Asian and Caucasian models when Chinese adults, brought up in Western countries, were made to see video clips of either Asian or Caucasian models receiving painful stimulations. Derntl et al. (2009) reported a decrease in amygdala activation in Asians who stayed longer in Europe during an explicit emotion recognition task involving Caucasian actors. Therefore, it is possible that exposure to multi-ethnic societies, including outgroups, generally reduced racial bias.

\section{Typical ethnicity and same-race faces when primed by ethnic context contrasted to cultural context}

Brain regions involved in social cognition, selfperception networks, and theory of mind networks were less activated in the ethnical context, than the cultural context. When out- and in-group faces of typical ethnicities (Chinese, Indian) were viewed in the ethnic context, contrasted to cultural contexts, higher activation of the FG was obtained, which is one of the core brain regions for face recognition (Tovée, 1995; Zhen et al., 2013). FG is commonly associated with in-group face processing (Guassi Moreira et al., 2016; Molenberghs, 2013; Shkurko, 2012; Van Bavel et al., 2008). However, there are studies that have reported, similarly, FG activations during out-group face processing (Ronquillo, 2010; Shkurko, 2010; lidaka, 2014). Although the MOG is involved in initial face processing as well (Pizzagalli et al., 2000), it also forms connections to higher social cognition areas such as the STS (Duvernoy \& Bourgouin, 1999; Jiang et al., 2018) and specialized cortices involved in emotional and value processing (Olson et al., 2013). Certain connections to higherlevel social cognitive areas may be due to the mixture of in-group Chinese and out-group Indian faces in typical ethnicities. A subset of similar regions was activated for in-group faces in the ethnic context.

However, same-race faces (in-group) in the cultural context, compared to ethnic context, elicited higher activation in mainly the MNS regions which are brain regions involved in social perception, and includes the VAA comprising of the lingual gyrus (Adolphs, 1999; Colton et al., 2013; Freeman et al., 2010; Scheepers et al., 2013), and the TPJ networks. The MNS system is famous for its activation while individuals observe the behavior of other people (Rizzolatti \& Craighero, 2004), and implicated in neurocognitive functions such as social cognition, empathy, and theory of mind (Rizzolatti \& Craighero, 2004; Sugranyes et al., 2011). TPJ is associated with the theory of mind and mentalizing (Freeman et al., 2010; Shkurko, 2012). Theory of mind refers to the 
process of explaining or predicting other people's behavior by way of reading, reasoning, and representing their independent mental states (Freeman et al., 2010). These regions are involved in self-other distinctions and making inferences about people (Greven \& Ramsey, 2017; Molenberghs, 2013). Consistent with the in-group findings, typical ethnicity faces in the cultural context, compared to ethnic context, elicited higher activations in regions associated with social perception and cognition (STS; $M T G, V A A$ to the lingual gyrus, along with the MNS and the PSA).

Interestingly, typical ethnicities and non-typical ethnicities were processed differently in both ethnic and cultural contexts. We did not observe any context effect when atypical other-race faces were viewed in the ethnic context, compared to the cultural context. Participants showed more sensitivity to contextual primes for typical ethnicities (Chinese and Indian faces) as opposed to non-typical ethnicities (Arab and Caucasian faces). One explanation is that our experimental design primarily tested the effect of a common shared culture between different ethnic groups, that also share common values of the belonging society. As a check on typical faces of different ethnicity, we added faces of people of diverse and non-typical ethnicity. In this sense, the results support the idea that manipulation of the context loses its meaning in the category of atypical ethnicity as there is no mutual cultural sharing.

Moreover, the present study showed that high social cognitive regions were recruited while viewing faces in the cultural context compared to more automatic and implicit processing of faces when viewed in their respective ethnic contexts. We found that viewing of in-group faces in the ethnic context showed typical in-group face brain activation compared to viewing them in the cultural context (i.e., higher activations in the FG (lidaka, 2014; Molenberghs, 2013; Van Bavel et al., 2008) and MOG (Jiang et al., 2018; Olson et al., 2013).

Overall, results indicate that in the cultural context, individuals engage higher social cognitive resources in order to make individuating judgments of people rather than superficial judgments based on group categorization (Freeman et al., 2010).

\section{Enlarged In-group Present in Cultural Context}

When neural responses to typical other- (outgroup) and same-race (in-group) faces were compared in the cultural context, no significant difference was found. Even using the less stringent $p$ value (uncorrected $p<0.0001$ ), the only difference was activation in the right $F G$ for in-group faces. In contrast, the difference between the out-group and ingroup faces in ethnic contexts was much more significant. Greater effort in social, cognitive, and affective processing was found for out-group faces in the ethnic context, as discussed earlier. As expected, the lack of difference in neural responses to outgroup (typical other-race) and in-group faces indicates that out-groups are processed similarly to in-groups. This phenomenon is characteristic of an enlarged cultural in-group. These findings are similar to the pattern of findings by Zuo, and Han (2013), who found no difference in neural activity in the pain matrix for in-group (Asians) and out-groups (Caucasians) amongst Chinese adults brought up in Western countries.

Specifically, the contextual manipulation of culture in this study may only be relevant to prime a common shared culture that is bound to exist only amongst well-integrated typical ethnicities, which may have rendered the contextual manipulation insensitive for non-typical ethnicities as seen in this study.

The analysis conducted to assess the difference in how neural responses differ for ingroups and out-groups based on context showed higher activation in the midline cortical regions for the cultural context compared to the ethnic context. This further suggests that the out-groups and in-groups may be processed similarly in the cultural context owing to individuating judgments of people rather than superficial judgments based on group categorization (Freeman et al., 2010), as suggested by high midline cortical activation in the cultural context.

Findings from this study suggest that identifying with a common shared culture, more than ethnicity, can drive a spontaneous sense of membership to a multi-ethnic group when the society explicitly and positively supports cross-ethnic interactions in every daily life context. These findings demonstrate that living in proximity and with an inclusive attitude with citizens from other ethnicities can expand the sense of membership of different individuals to that of a member of the same cultural group. The results shed light on a possible emergent phenomenon of a new way to categorize oneself and others, in terms of membership, which stands between the classical 
ethnic in- and out-group categorization and where culture and not ethnicity shapes and drives spontaneous social judgment of others (Figure 5).

However, further research can also be extended to investigating brain reactivity and responsiveness to different ethnic baby faces in a multicultural society and the effect of the environmental context. Such insight will give a substantial theoretical contribution to building an integrated and general model of the social brain.

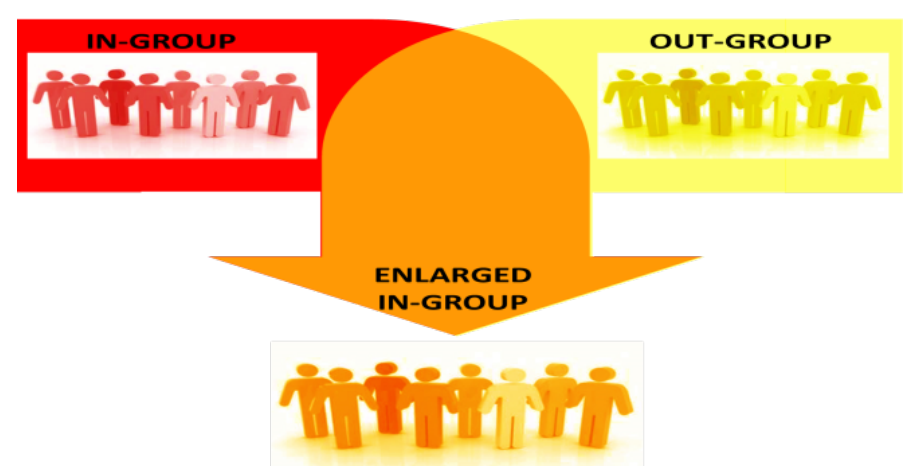

Figure 5. Graphical representation of a likely scenario of in- and out-group membership dynamics in Singapore. Red and yellow colors are namely ethnic-based in-groups and out-groups; orange color is the new phenomenon of the culture-based enlarged in-group which integrates features from both of the above mentioned groups.

\section{Limitations}

Firstly, only the majority ethnicity of Singapore (Chinese) participants participated in the study. However, majority and minority groups may perceive and experience intergroup relations differently following their experiences (Dovidio et al., 2008). In order to get a holistic understanding, neural responses to enlarged cultural in-groups should be investigated amongst minority groups as well.

Another limitation is the sample of the age group. All participants were young adults (students or recently graduated). Age differences were reported in studies involving in-group and out-group (Guassi Moreira et al., 2016) and social cognition areas (Colten et al., 2013). Therefore, the effect of enlarged cultural ingroup is necessary to be investigated across generations to generalize the findings wholly.

Posing another limitation is that the face stimuli used in this study are only of the female gender. Research from intergroup relations has shown that gender influences in-group racial bias (e.g., Navarrete et al., 2010; Rudman \& Goodwin, 2004). Face stimuli should be both male and female in order to analyze any gender effects.

Lastly, all the faces were unfamiliar to participants.

Familiar and new faces have differing neural activation. Intergroup friendships are common in multicultural societies. Therefore, it is necessary to understand the neural representation of familiar faces in ethnic in-group, culturally enlarged in-group, and out-group faces.

\section{Conclusion}

Although findings from this study come with certain limitations, it is the first step towards integrating and constructing a model of the multicultural social brain. Building and identifying with a common shared culture in multicultural societies enhance the individuating processing of ethnic outgroups and leads to the formation of an enlarged cultural multi-ethnic in-group. In a well-integrated multicultural society, members do not innately respond to other ethnicities with typical out-group bias responses, but dispense more social and cognitive resources into perceiving them more as individuals than superficially as an out-group. These pioneering findings of the existence of an enlarged cultural ingroup open the opportunity for future research to develop on the understanding of multicultural societies and its complex interaction with contextual environment towards dynamic perceptions of ingroups and out-groups through a multi-level approach.

\section{Acknowledgements}

All participants in this study are gratefully acknowledged. The authors thank Lim Mengyu for assistance. This research was supported by NAP SUG 2015 (GE) and Singapore Ministry of Education ACR Tier 1 (GE; RG149/16 and RT10/19).

\section{References}

Adolphs, R. (1999). Social cognition and the human brain. Trends in cognitive sciences, 3(12), 469-479.

Adolphs, R. (2001). The neurobiology of social cognition. Current opinion in neurobiology, 11(2), 231-239.

Adolphs, R., Tranel, D., \& Damasio, A. R. (1998). The human amygdala in social judgment. Nature, 393(6684), 470.

Ålund, A. (1999). Ethnicity, multiculturalism and the problem of culture. European Societies, 1(1), 105-116.

Allan, H. T. (2008). Ethnicity, race and health in a multicultural environment: foundations for better epidemiology, public health 
bioRxiv preprint doi: https://doi.org/10.1101/2020.06.12.148601; this version posted June 13,2020 . The copyright holder for this preprint (which was not certified by peer review) is the author/funder, who has granted bioRxiv a license to display the preprint in perpetuity. It is made available under aCC-BY-NC 4.0 International license.

and health care. Bulletin of the World Health Organization, 86(3), 238-238.

Bernhardt, B. C., \& Singer, T. (2012). The neural basis of empathy. Annual review of neuroscience, 35, 1-23.

Bollinger, J., Rubens, M. T., Zanto, T. P., \& Gazzaley, A. (2010). Expectation-driven changes in cortical functional connectivity influence working memory and long-term memory performance. Journal of Neuroscience, 30(43), 14399-14410.

Candidi, M., Stienen, B. M., Aglioti, S. M., \& de Gelder, B. (2015). Virtual lesion of right posterior superior temporal sulcus modulates conscious visual perception of fearful expressions in faces and bodies. Cortex, 65, 184-194.

Caria, A., de Falco, S., Venuti, P., Lee, S., Esposito, G., Rigo, P., Birbaumer, N., \& Bornstein, M.H. (2012) Species-specific response to human infant faces in the premotor cortex. Neuroimage, 60(2):884-893.

Chan, A. W. Y., \& Baker, C. I. (2015). Seeing is not feeling: posterior parietal but not somatosensory cortex engagement during touch observation. Journal of Neuroscience, 35(4), 14681480.

Cheon, B., \& Esposito, G. (2020). Brief Exposure to Infants Activates Social and Intergroup Vigilance. Behavioral Sciences, 10, 72.

Colton, G., Leshikar, E. D., \& Gutchess, A. H. (2013). Age differences in neural response to stereotype threat and resiliency for self-referenced information. Frontiers in human neuroscience, 7, 537.

Crisp, R. J., \& Hewstone, M. (1999). Differential evaluation of crossed category groups: Patterns, processes, and reducing intergroup bias. Group Processes \& Intergroup Relations, 2(4), 307-333.

Cunningham, W. A., Johnson, M. K., Raye, C. L., Gatenby, J. C., Gore, J. C., \& Banaji, M. R. (2004). Separable neural components in the processing of black and white faces. Psychological Science, 15(12), 806-813.

Cunningham, W., Van Bavel, J., Arbuckle, N., Packer, D., \& Waggoner, A. (2012). Rapid social perception is flexible: approach and avoidance motivational states shape $\mathrm{P} 100$ responses to other-race faces. Frontiers in Human Neuroscience, 6, 140.

Derks, B., Stedehouder, J., \& Ito, T. A. (2014). Social identity modifies face perception: An ERP study of social categorization. Social cognitive and affective neuroscience, 10(5), 672-679.

Derntl, B., Habel, U., Robinson, S., Windischberger, C., Kryspin-Exner, I., Gur, R. C., \& Moser, E. (2009). Amygdala activation during recognition of emotions in a foreign ethnic group is associated with duration of stay. Social Neuroscience, 4(4), 294-307.

Dijkstra, S., Geuijen, K., \& De Ruijter, A. (2001). Multiculturalism and social integration in Europe. International Political Science Review, 22(1), 55-84.

Dorland, W. A. N. (2011). Dorland's Illustrated Medical Dictionary32: Dorland's Illustrated Medical Dictionary. Elsevier Health Sciences 1327.

Dovidio, J. F., Gaertner, S. L., \& Saguy, T. (2008). Another view of "we": Majority and minority group perspectives on a common ingroup identity. European review of social psychology, 18(1), 296-330.
Duvernoy, H. M., \& Bourgouin, P. (1999). The human brain: Surface, three-dimensional sectional anatomy with MRI, and blood supply. Wien: Springer.

Elfenbein, H. A., \& Ambady, N. (2002). On the universality and cultural specificity of emotion recognition: a metaanalysis. Psychological bulletin, 128(2), 203.

Esposito, G., Nakazawa, J., Ogawa, S., Stival, R., Kawashima, A., Putnick, D.L., \& Bornstein, M.H. (2014). Baby, You Light-up My Face: Culture-General Physiological Responses to Infants and Culture-Specific Cognitive Judgements of Adults. PlosONE, 9(10):e106705.

Esposito G., Nakazawa J., Ogawa S., Stival R., Putnick D.L., \& Bornstein MH. (2015). Using Infrared Thermography to Assess Emotional Responses to Infants. Early Child Development and Care, 185(3), 438-447.

Falk, E. B., Spunt, R. P., \& Lieberman, M. D. (2012). Ascribing beliefs to ingroup and outgroup political candidates: neural correlates of perspective-taking, issue importance and days until the election. Phil. Trans. R. Soc. B, 367(1589), 731-743.

Freeman, J. B., Schiller, D., Rule, N. O., \& Ambady, N. (2010). The neural origins of superficial and individuated judgments about ingroup and outgroup members. Human brain mapping, 31(1), 150-159.

Gaertner, S. L., \& Dovidio, J. F. (2014). Reducing intergroup bias: The common ingroup identity model. Psychology Press.

Gijsberts, M., Van Der Meer, T., \& Dagevos, J. (2011). 'Hunkering down'in multi-ethnic neighbourhoods? The effects of ethnic diversity on dimensions of social cohesion. European Sociological Review, 28(4), 527-537.

Gobbini, M. I., \& Haxby, J. V. (2006). Neural response to the visual familiarity of faces. Brain research bulletin, 71(1-3), 76-82.

Gobbini, M. I., Leibenluft, E., Santiago, N., \& Haxby, J. V. (2004). Social and emotional attachment in the neural representation of faces. Neuroimage, 22(4), 1628-1635.

Golby, A. J., Gabrieli, J. D., Chiao, J. Y., \& Eberhardt, J. L. (2001). Differential responses in the fusiform region to same-race and other-race faces. Nature neuroscience, 4(8), 845.

Green, S. A., Hernandez, L. M., Bowman, H. C., Bookheimer, S. Y., \& Dapretto, M. (2018). Sensory over-responsivity and social cognition in ASD: Effects of aversive sensory stimuli and attentional modulation on neural responses to social cues. Developmental cognitive neuroscience, 29, 127-139.

Greven, I. M., \& Ramsey, R. (2017). Neural network integration during the perception of in-group and out-group members. Neuropsychologia, 106, 225-235.

Guassi Moreira, J. F., Van Bavel, J. J., \& Telzer, E. H. (2016). The neural development of 'us and them'. Social cognitive and affective neuroscience, 12(2), 184-196.

Harris, L. T., \& Fiske, S. T. (2006). Dehumanizing the lowest of the low: Neuroimaging responses to extreme outgroups. Psychological science, 17(10), 847-853.

Han, S. (2015). Intergroup relationship and empathy for others' pain: a social neuroscience approach. In Neuroscience in Intercultural Contexts (pp. 31-47). Springer, New York, NY.

Hehman, E., Stanley, E. M., Gaertner, S. L., \& Simons, R. F. (2011). Multiple group membership influences face-recognition: Recall and neurological evidence. Journal of Experimental Social Psychology, 47(6), 1262-1268.

Holmes, A. P., \& Friston, K. J. (1998). Generalisability, random E ects \& population inference. Neuroimage, 7, S754. 
bioRxiv preprint doi: https://doi.org/10.1101/2020.06.12.148601; this version posted June 13,2020 . The copyright holder for this preprint (which was not certified by peer review) is the author/funder, who has granted bioRxiv a license to display the preprint in perpetuity. It is made available under aCC-BY-NC 4.0 International license.

Holtug, N. (2016). Multiculturalism and Social Cohesion. In Migration and Integration. New Models for Mobility and Coexistence (pp. 71-84). Vienna University Press

Housing \& Development Board. (2017, Nov 23). Public Housing - A Singapore Icon.

Retrieved from http://www.hdb.gov.sg/cs/infoweb/aboutus/our-role/public-housing--

a-singapore-icon

Hugenberg, K., \& Wilson, J. P. (2013). Faces are central to social cognition. Handbook of social cognition, 167-193.

lidaka, T. (2012). The role of the superior temporal sulcus in face recognition and perception. Brain and nerve= Shinkei kenkyu no shinpo, 64(7), 737-742.

lidaka, T. (2014). Role of the fusiform gyrus and superior temporal sulcus in face perception and recognition: An empirical review. Japanese Psychological Research, 56(1), 33-45.

Igelström, K. M., \& Graziano, M. S. (2017). The inferior parietal lobule and temporoparietal junction: a network perspective. Neuropsychologia, 105, 70-83.

Ionta, S., Martuzzi, R., Salomon, R., \& Blanke, O. (2014). The brain network reflecting bodily self-consciousness: a functional connectivity study. Social cognitive and affective neuroscience, 9(12), 1904-1913.

Ito, T. A., \& Urland, G. R. (2003). Race and gender on the brain: electrocortical measures of attention to the race and gender of multiply categorizable individuals. Journal of personality and social psychology, 85(4), 616.

Jiang, X., Sanford, R., \& Pell, M. D. (2018). Neural architecture underlying person perception from in-group and outgroup voices. Neurolmage, 181, 582-597.

Kaplan, J. T., Freedman, J., \& lacoboni, M. (2007). Us versus them: Political attitudes and party affiliation influence neural response to faces of presidential candidates. Neuropsychologia, 45(1), 55-64.

Katsumi, Y., \& Dolcos, S. (2018). Neural Correlates of Racial Ingroup Bias in Observing Computer-Animated Social Encounters. Frontiers in human neuroscience, 11, 632.

Kaul, C., Ratner, K. G., \& Van Bavel, J. J. (2012). Dynamic representations of race: processing goals shape race decoding in the fusiform gyri. Social cognitive and affective neuroscience, 9(3), 326-332.

Kærgård, N. (2010). Social cohesion and the transformation from ethnic to multicultural society: The case of Denmark. Ethnicities, 10(4), 470-487.

Kearns, A., \& Whitley, E. (2018). Perceived neighborhood ethnic diversity and social outcomes: Context-dependent effects within a postindustrial city undergoing regeneration. Journal of urban affairs, 40(2), 186-208.

Keong, C. S. (2013). Multiculturalism in Singapore-The Way to a Harmonious Society. SACLJ, 25, 84.

Keysers, C., Kaas, J. H., \& Gazzola, V. (2010). Somatosensation in social perception. Nature Reviews Neuroscience, 11(6), 417.

Knutson, K. M., Mah, L., Manly, C. F., \& Grafman, J. (2007). Neural correlates of automatic beliefs about gender and race. Human brain mapping, 28(10), 915-930.

Kurzban, R., Tooby, J., \& Cosmides, L. (2001). Can race be erased? Coalitional computation and social categorization. Proceedings of the National Academy of Sciences, 98(26), 15387-15392.
Lim, J. (2010). Maintenance of Religious Harmony Bill. NLB Singapore Infopedia. Retrieved

from

http://eresources.nlb.gov.sg/infopedia/articles/SIP_1638_201001-31.html

Logan, B. R., \& Rowe, D. B. (2004). An evaluation of thresholding techniques in fMRI analysis. Neurolmage, 22(1), 95108.

Mahmud, A., \& Jahan, H. (2013). Social Cohesion in Multicultural Society: A Case of Bangladeshi Immigrants in Stockholm.

Mathews, M., Lim, L., Shanthini, S., \& Cheung, N. (2017). CNA-IPS SURVEY ON ETHNIC IDENTITY IN SINGAPORE. Retrieved from http://lkyspp2.nus.edu.sg/ips/wpcontent/uploads/sites/2/2017/11/I PS-Working-Paper-28_081117.pdf

Molenberghs, P. (2013). The neuroscience of in-group bias. Neuroscience \& Biobehavioral Reviews, 37(8), 1530-1536.

National Library Board. (2013, Oct 23). Group representation constituencies. Retrieved from

http://eresources.nlb.gov.sg/infopedia/articles/SIP_2013-10-

25_182746.html

National Library Board. (2014, Sept 29). Racial Harmony Day.

Retrieved from

http://eresources.nlb.gov.sg/infopedia/articles/SIP_965_200412-31.html

Olson, I. R., McCoy, D., Klobusicky, E., \& Ross, L. A. (2013). Social cognition and the anterior temporal lobes: a review and theoretical framework. Social cognitive and affective neuroscience, 8(2), 123-133.

Phelps, E. A., O'Connor, K. J., Cunningham, W. A., Funayama, E. S., Gatenby, J. C., Gore, J. C., \& Banaji, M. R. (2000). Performance on indirect measures of race evaluation predicts amygdala activation. Journal of cognitive neuroscience, 12(5), 729-738.

Pitcher, D. (2014). Facial expression recognition takes longer in the posterior superior temporal sulcus than in the occipital face area. Journal of Neuroscience, 34(27), 9173-9177.

Pizzagalli, D., Lehmann, D., König, T., Regard, M., \& PascualMarqui, R. D. (2000). Face-elicited ERPs and affective attitude: brain electric microstate and tomography analyses. Clinical Neurophysiology, 111(3), 521-531.

Platek, S. M., \& Kemp, S. M. (2009). Is family special to the brain? An event-related fMRI study of familiar, familial, and selfface recognition. Neuropsychologia, 47(3), 849-858.

Platek, S. M., Loughead, J. W., Gur, R. C., Busch, S., Ruparel, K., Phend, N., Panyavin, I.S., \& Langleben, D. D. (2006). Neural substrates for functionally discriminating self-face from personally familiar faces. Human brain mapping, 27(2), 91-98.

Quadflieg, S., Turk, D. J., Waiter, G. D., Mitchell, J. P., Jenkins, A. C., \& Macrae, C. N. (2009). Exploring the neural correlates of social stereotyping. Journal of Cognitive Neuroscience, 21(8), 1560-1570.

Pourtois, G., Sander, D., Andres, M., Grandjean, D., Reveret, L., Olivier, E., \& Vuilleumier, P. (2004). Dissociable roles of the human somatosensory and superior temporal cortices for processing social face signals. European Journal of Neuroscience, 20(12), 3507-3515.

Rizzolatti, G., \& Craighero, L. (2004). The mirror-neuron system. Annu. Rev. Neurosci., 27, 169-192. 
Richeson, J. A., \& Shelton, J. N. (2003). When prejudice does not pay: Effects of interracial contact on executive function. Psychological Science, 14(3), 287-290.

Ronquillo, J. M. (2010). Neural correlates of learning and reversal of approach versus withdraw responses to in-and outgroup individuals. University of Southern California.

Rosen, M. L., Sheridan, M. A., Sambrook, K. A., Peverill, M. R., Meltzoff, A. N., \& McLaughlin, K. A. (2018). The role of visual association cortex in associative memory formation across development. Journal of cognitive neuroscience, 30(3), 365-380.

Rosenthal, L., \& Levy, S. R. (2010). The colorblind, multicultural, and polycultural ideological approaches to improving intergroup attitudes and relations. Social Issues and Policy Review, 4(1), 215-246.

Salleh, N. M. (2017, May 20). Singaporeans have evolved a distinctive identity: PM Lee Hsien Loong. The Straits Times (Singapore). Retrieved from https://www.straitstimes.com/singapore/singaporeans-haveevolved-a-distinctive-identity-pm

Schaefer, M., Heinze, H. J., \& Rotte, M. (2013). Interaction of embodied cognition and social perception in the primary somatosensory cortex. Multisensory Research, 26, 102-102.

Scheepers, D., Derks, B., Nieuwenhuis, S., Lelieveld, G. J., Van Nunspeet, F., Rombouts, S. A., \& De Rover, M. (2013). The neural correlates of in-group and self-face perception: is there overlap for high identifiers?. Frontiers in human neuroscience, 7 , 528.

Schobert, A. K., Corradi-Dell'Acqua, C., Frühholz, S., van der Zwaag, W., \& Vuilleumier, P. (2017). Functional organization of face processing in the human superior temporal sulcus: a $7 \mathrm{~T}$ high-resolution fMRI study. Social cognitive and affective neuroscience, 13(1), 102-113.

Schurz, M., Tholen, M. G., Perner, J., Mars, R. B., \& Sallet, J. (2017). Specifying the brain anatomy underlying temporo-parietal junction activations for theory of mind: A review using probabilistic atlases from different imaging modalities. Human brain mapping, 38(9), 4788-4805.

Semrud-Clikeman, M., Fine, J. G., \& Zhu, D. C. (2011). The role of the right hemisphere for processing of social interactions in normal adults using functional magnetic resonance imaging. Neuropsychobiology, 64(1), 47-51.

Serino, A., Giovagnoli, G., \& Làdavas, E. (2009). I feel what you feel if you are similar to me. PloS one, 4(3), e4930.

Shkurko, A. V. (2012). Is social categorization based on relational ingroup/outgroup opposition? A meta-analysis. Social cognitive and affective neuroscience, 8(8), 870-877.

Soto, J. A., \& Levenson, R. W. (2009). Emotion recognition across cultures: The influence of ethnicity on empathic accuracy and physiological linkage. Emotion, 9(6), 874.

StraitsTimes. (2017, May 19). Singapore's approach to diversity has created a distinctive identity across ethnic groups:
PM Lee Hsien Loong. The Straits Times (Politics). Retrieved from https://www.straitstimes.com/politics/singapolitics/pm-whetherchinese-malay-or-indian-a-singaporean-can-spot-a-fellow-citizen

Sturm, V. E., Haase, C. M., \& Levenson, R. W. (2016). Emotional Dysfunction in Psychopathology and Neuropathology: Neural and Genetic Pathways. In Genomics, Circuits, and Pathways in Clinical Neuropsychiatry (pp. 345-364).

Sugranyes, G., Kyriakopoulos, M., Corrigall, R., Taylor, E., \& Frangou, S. (2011). Autism spectrum disorders and schizophrenia: meta-analysis of the neural correlates of social cognition. Plos one, 6(10), e25322.

Tovée, M. J. (1995). Face Recognition: What are faces for?. Current Biology, 5(5), 480-482.

Turner, J. C., Oakes, P. J., Haslam, S. A., \& McGarty, C. (1994). Self and collective: Cognition and social context. Personality and social psychology bulletin, 20(5), 454463.

Valchev, N., Gazzola, V., Avenanti, A., \& Keysers, C. (2016). Primary somatosensory contribution to action observation brain activity-combining $\mathrm{FMRI}$ and cTBS. Social cognitive and affective neuroscience, 11(8), 1205-1217.

Van Bavel, J. J., \& Cunningham, W. A. (2010). A social neuroscience approach to self and social categorisation: A new look at an old issue. European Review of Social Psychology, 21(1), 237-284.

Van Bavel, J. J., Packer, D. J., \& Cunningham, W. A. (2008). The neural substrates of in-group bias: a functional magnetic resonance imaging investigation. Psychological science, 19(11), 1131-1139.

Venturoso, L., Gabrieli, G., Truzzi, A., Azhari, A., Setoh, P., Bornstein, M.H., Esposito, G. (2019) Effects of Baby Schema and Mere Exposure on Explicit and Implicit Face Processing. Frontiers in Psychology, 10:2649.

Webb, W. G. (2017). Neurosensory Organization. Neurology for the Speech-Language Pathologist,93-109. doi:10.1016/b9780-323-10027-4.00005-1

Wheeler, M. E., \& Fiske, S. T. (2005). Controlling racial prejudice: Social-cognitive goals affect amygdala and stereotype activation. Psychological Science, 16(1), 56-63.

Weller, J. A., Levin, I. P., Shiv, B., \& Bechara, A. (2009). The effects of insula damage on decision-making for risky gains and losses. Social neuroscience, 4(4), 347-358.

Whalen, P. J. (1998). Fear, vigilance, and ambiguity: Initial neuroimaging studies of the human amygdala. Current directions in psychological science, 7(6), 177-188.

Zhen, Z., Fang, H., \& Liu, J. (2013). The hierarchical brain network for face recognition. PloS one, 8(3), e59886.

Zuo, X., \& Han, S. (2013). Cultural experiences reduce racial bias in neural responses to others' suffering. Culture and Brain, 1(1), 34-46. 
Table 1. Sites of significant neural activation ( $p<0.05$, FWE correction) related to the contrasts typical faces in CulCon vs. typical faces in EthCon and non-typical faces in CulCon vs. non-typical faces in EthCon.

\begin{tabular}{|c|c|c|c|c|c|c|c|c|c|c|}
\hline \multirow{2}{*}{\multicolumn{3}{|c|}{ Comparison of Interest }} & \multirow{2}{*}{ Brain Regions } & \multirow{2}{*}{ BA } & \multirow{2}{*}{ Left/Right } & \multicolumn{3}{|c|}{ MNI Coordinates } & \multirow{2}{*}{$\begin{array}{l}\text { Voxels at } \\
\text { p(FWE)<.05 }\end{array}$} & \multirow{2}{*}{ MeanT } \\
\hline & & & & & & $\mathbf{x}$ & $y$ & $z$ & & \\
\hline \multirow[t]{6}{*}{$\begin{array}{l}\text { typical faces in } \\
\text { CulCon }\end{array}$} & $>$ & $\begin{array}{l}\text { typical faces in } \\
\text { EthCon }\end{array}$ & Superior Temporal Gyrus & 22 & Right & 60 & -7 & 8 & 53 & 5.3192 \\
\hline & & & Supramarginal gyrus & 40 & Right & 48 & -31 & 20 & 17 & 5.3207 \\
\hline & & & Visual Association Area & 18 & Right & 12 & -94 & 20 & 34 & 5.2318 \\
\hline & & & $\begin{array}{l}\text { Visual Association Area/Lingual } \\
\text { gyrus }\end{array}$ & 18 & Left & -9 & -70 & -4 & 12 & 5.6930 \\
\hline & & & Middle Temporal gyrus & 21 & Left & -63 & -37 & 8 & 24 & 5.1921 \\
\hline & & & $\begin{array}{l}\text { Primary Sensory Area/Postcentral } \\
\text { gyrus }\end{array}$ & 1 & Right & 27 & -40 & 62 & 24 & 5.1568 \\
\hline \multirow[t]{5}{*}{$\begin{array}{l}\text { typical faces in } \\
\text { EthCon }\end{array}$} & $>$ & $\begin{array}{l}\text { typical faces in } \\
\text { CulCon }\end{array}$ & Fusiform gyrus & 37 & Right & 30 & -46 & -13 & 104 & 6.4733 \\
\hline & & & Middle Occipital gyrus & 19 & Right & 33 & -76 & 20 & 70 & 6.1197 \\
\hline & & & Fusiform area & 37 & Left & -33 & -49 & -13 & 59 & 5.9118 \\
\hline & & & Inferior frontal gyrus & 44 & Right & 42 & 8 & 32 & 30 & 5.6794 \\
\hline & & & Middle Occipital gyrus & 19 & Left & -27 & -85 & 17 & 71 & 5.6935 \\
\hline
\end{tabular}




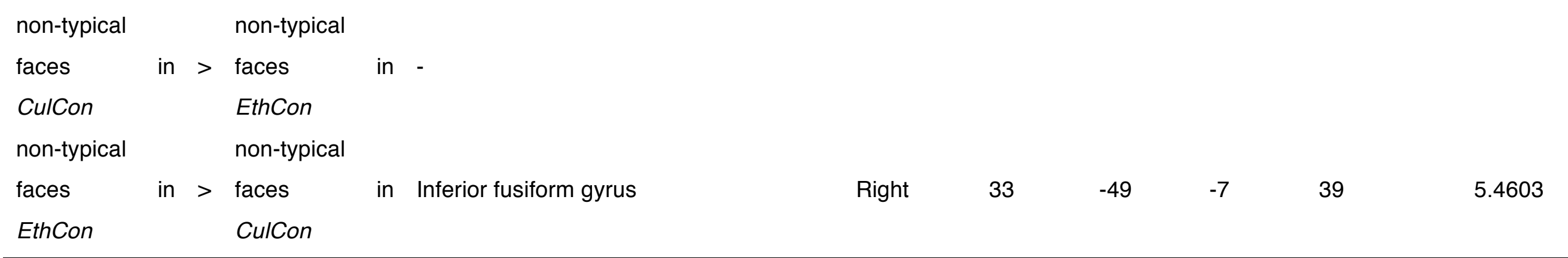


Table 2. Sites of significant neural activation ( $\mathrm{p}<0.05$, FWE correction) related to the IF in CulCon vs. IF in EthCon

\begin{tabular}{|c|c|c|c|c|c|c|c|c|c|}
\hline \multirow{2}{*}{\multicolumn{2}{|c|}{ Comparison of Interest }} & \multirow{2}{*}{ Brain Regions } & \multirow{2}{*}{ BA } & \multirow{2}{*}{ Left/Right } & \multicolumn{3}{|c|}{ MNI Coordinates } & \multirow{2}{*}{$\begin{array}{l}\text { Voxels at } \\
p(F W E)<.05\end{array}$} & \multirow{2}{*}{ MeanT } \\
\hline & & & & & $\mathbf{x}$ & $y$ & $z$ & & \\
\hline \multirow[t]{3}{*}{ IF in CulCon } & $>\quad I F$ in EthCon & $\begin{array}{l}\text { Primary Sensory area/Postcentral } \\
\text { gyrus }\end{array}$ & 1 & Right & 33 & -22 & 44 & 160 & 5.3023 \\
\hline & & Rolandic Operculum & & Right & 51 & -16 & 11 & 102 & 5.3401 \\
\hline & & $\begin{array}{l}\text { Visual association area/Lingual } \\
\text { gyrus }\end{array}$ & 18 & Right & 9 & -70 & -1 & 11 & 5.3043 \\
\hline
\end{tabular}


Cuneous

IF in EthCon $>\quad I F$ in CulCon

\section{Inferior fusiform gyrus}

Posterior fusiform gyrus

Middle occipital gyrus
Right

Right

Right

Right
12

$-82$

$33-52$

33

30

33
26

$-10$

$-7$

20
37

25

17

13
5.3215

5.7091

5.5064

5.4532 
Table 3. Sites of significant neural activation ( $p<0.001$, uncorrected) related to the contrast OF in CulCon vs. OF in EthCon.

\begin{tabular}{|c|c|c|c|c|c|c|c|c|c|}
\hline \multirow{2}{*}{\multicolumn{2}{|c|}{ Comparison of Interest }} & \multirow{2}{*}{ Brain Regions } & \multirow{3}{*}{ BA } & \multirow{3}{*}{ Left/Right } & \multicolumn{3}{|c|}{ MNI Coordinates } & \multirow{2}{*}{$\begin{array}{l}\text { Voxels at } \\
p \text { (uncorr)<.0001 }\end{array}$} & \multirow{2}{*}{ MeanT } \\
\hline & & & & & $\mathbf{x}$ & $\mathbf{y}$ & $\mathbf{z}$ & & \\
\hline OF in CulCon & $>O F$ in EthCon & No voxles survied threshold & & & & & & & \\
\hline \multirow[t]{4}{*}{ OF in EthCon } & $>O F$ in CulCon & Fusiform & & Right & 33 & -46 & -7 & 107 & 3.9091 \\
\hline & & Lingual & & Left & -24 & -64 & -7 & 74 & 3.7296 \\
\hline & & Visual association area & 18 & Right & 15 & -73 & -1 & 23 & 3.5755 \\
\hline & & Postcentral gyrus & & Left & -39 & -19 & 26 & 18 & 3.4173 \\
\hline
\end{tabular}


Table 4. Sites of significant neural activation related to the contrast OF in CulCon vs. IF in CulCon.

\begin{tabular}{|c|c|c|c|c|c|c|c|c|c|}
\hline \multirow{2}{*}{\multicolumn{2}{|c|}{ Comparison of Interest }} & \multirow{2}{*}{ Brain Regions } & \multirow{2}{*}{ BA } & \multirow{2}{*}{ Left/Right } & \multicolumn{3}{|c|}{ MNI Coordinates } & \multirow{2}{*}{ Voxels } & \multirow{2}{*}{ MeanT } \\
\hline & & & & & $\mathbf{x}$ & y & $\mathbf{z}$ & & \\
\hline \multicolumn{10}{|l|}{$p(F W E)<0.05$} \\
\hline IF in CulCon & $>$ OF in CulCon & - & & & & & & & \\
\hline OF in CulCon & $>I F$ in CulCon & - & & & & & & & \\
\hline \multicolumn{10}{|c|}{$p$ (uncorr) $<0.0001$} \\
\hline IF in CulCon & $>$ OF in CulCon & Fusiform gyrus & & Right & 33 & -46 & -7 & 42 & 4.5133 \\
\hline OF in CulCon & $>I F$ in CulCon & - & & & & & & & \\
\hline
\end{tabular}


Table 5. Sites of significant neural activation $(\mathrm{p}<0.05$, FWE) related to the contrast $O F$ in EthCon vs. IF in EthCon.

\begin{tabular}{|c|c|c|c|c|c|c|c|c|c|}
\hline \multirow{2}{*}{\multicolumn{2}{|c|}{ Comparison of Interest }} & \multirow{3}{*}{$\begin{array}{l}\text { Brain Regions } \\
\text { Fusiform area }\end{array}$} & \multirow{3}{*}{ BA } & \multirow{3}{*}{$\begin{array}{l}\text { Left/Right } \\
\text { Right }\end{array}$} & \multicolumn{3}{|c|}{ MNI Coordinates } & \multirow{2}{*}{$\begin{array}{l}\text { Voxels at } \\
p(F W E)<.05\end{array}$} & \multirow{2}{*}{ MeanT } \\
\hline & & & & & $\mathbf{x}$ & $\mathbf{y}$ & $\mathbf{z}$ & & \\
\hline IF in EthCon & $>$ OF in EthCon & & & & 33 & -52 & -10 & 35 & 5.4098 \\
\hline \multirow[t]{9}{*}{ OF in EthCon } & $>$ IF in EthCon & $\begin{array}{l}\text { Visual association area/Lingual } \\
\text { gyrus }\end{array}$ & 18 & Right & 9 & -70 & -1 & 32 & 5.4935 \\
\hline & & Lingual gyrus & & Left & -9 & -64 & -7 & 27 & 5.5316 \\
\hline & & Sensory Association area & 5 & Right & 21 & -43 & 62 & 98 & 5.2934 \\
\hline & & Rolandic Operculum & & Right & 51 & -31 & 20 & 89 & 5.2849 \\
\hline & & Cuneus & & Right & 12 & -82 & 29 & 34 & 5.3759 \\
\hline & & Rolandic Operculum & & Left & -39 & -19 & 23 & 10 & 5.2938 \\
\hline & & Rolandic Operculum & 6 & Right & 54 & -4 & 5 & 21 & 5.2983 \\
\hline & & Superior Temporal gyrus & & Left & -57 & -25 & 11 & 18 & 5.2113 \\
\hline & & Supramarginal gyrus & 40 & Left & -60 & -34 & 29 & 12 & 5.1392 \\
\hline
\end{tabular}


Table 6. Sites of significant neural activation $(\mathrm{p}<0.05$, FWE) related to the contrast IF in CulCon $>$ OF in CulCon vs. IF EthCon $>$ OF in EthCon.

\begin{tabular}{|c|c|c|c|c|c|c|c|c|c|}
\hline \multirow{2}{*}{\multicolumn{2}{|c|}{ Comparison of Interest }} & \multirow{3}{*}{$\begin{array}{l}\text { Brain Regions } \\
\text { Lingual gyrus }\end{array}$} & \multirow{3}{*}{ BA } & \multirow{3}{*}{$\begin{array}{l}\text { Left/Right } \\
\text { Right }\end{array}$} & \multicolumn{3}{|c|}{ MNI Coordinates } & \multirow{3}{*}{$\begin{array}{l}\text { Voxels at } \\
q(F W E)<.05 \\
42\end{array}$} & \multirow{3}{*}{$\begin{array}{r}\text { MeanT } \\
5.3767\end{array}$} \\
\hline & & & & & \multirow{2}{*}{$\frac{x}{9}$} & \multirow{2}{*}{$\frac{y}{-70}$} & \multirow{2}{*}{$\begin{array}{l}z \\
-1\end{array}$} & & \\
\hline $\begin{array}{l}\text { IF in CulCon }> \\
\text { OF in CulCon }\end{array}$ & $\begin{array}{l}\text { IF in EthCon > } \\
\text { OF in EthCon }\end{array}$ & & & & & & & & \\
\hline & & Postcentral gyrus/Precuneus & 7 & Right & 18 & -45 & 65 & 73 & 4.9517 \\
\hline & & Cuneus & & Right & 12 & -85 & 29 & 34 & 5.0007 \\
\hline & & Lingual gyrus & & Left & -9 & -73 & -4 & 10 & 4.9263 \\
\hline & & Precentral gyrus & & Left & -36 & -28 & 62 & 29 & 4.8493 \\
\hline $\begin{array}{l}\text { IF in EthCon > } \\
\text { OF in EthCon }\end{array}$ & $\begin{array}{l}\text { IF in CulCon > } \\
\text { OF in CulCon }\end{array}$ & - & & & & & & & \\
\hline \multirow{2}{*}{\multicolumn{2}{|c|}{ Conjuction analysis }} & Fusiform & & Right & 33 & -52 & -10 & 132 & 5.8720 \\
\hline & & Fusiform & & Left & -30 & -43 & -13 & 36 & 5.1782 \\
\hline
\end{tabular}

